\title{
Categorização de vídeos educacionais do Youtube por meio de comentários
}

\author{
Henrique Carlos Fonte Boa Carvalho - UFVJM - henriquefbc@ gmail.com \\ Cristiano Grijó Pitangui - UFSJ - pitangui.cristiano@gmail.com \\ Eduardo Augusto Costa Trindade - UFVJM - eduardo.trindade@ufvjm.edu.br \\ Luciana Pereira de Assis - UFVJM - lpassis@ufvjm.edu.br \\ Alessandro Vivas Andrade - UFVJM - alessandrovivas@ufvjm.edu.br \\ Dayane Priscila Bicalho de Souza - FAVENI - dayanepriscila90@hotmail.com
}

\begin{abstract}
Resumo: O Youtube é uma plataforma online que oferta Objetos de Aprendizagem sobre as mais diversas áreas do conhecimento. De maneira a facilitar a busca por conteúdos, o Youtube categoriza os seus vídeos em diversas classes. No entanto, já apontou-se que, de forma geral, a categorização dos vídeos não é relevante ou que as categorias estão erroneamente atribuídas aos vídeos. Este trabalho utiliza técnicas de Mineração de Texto com o objetivo de analisar os comentários dos vídeos e identificar se eles possuem potencial para serem utilizados para a categorização de vídeos educacionais. Resultados experimentais apontam que existem diferenças importantes nos vocabulários dos comentários dos vídeos educacionais e não educacionais.
\end{abstract}

Palavras-chave: categorização, mineração de texto, youtube.

\section{Categorization of Youtube's educational videos through comments}

\begin{abstract}
Youtube is an online sharing video platform that offers Learning Objects on several knowledge areas. In order to facilitate the content search, Youtube categorizes its videos in different classes. However, it has already been pointed out that, in general, this categorization is not relevant or that the categories are erroneously assigned to videos. This work uses Text Mining techniques in order to analyze the comments of the videos and identify if they have potential to be used in order to categorize educational videos. Experimental results indicate that there are important differences in the vocabularies of the comments of educational and non-educational videos.
\end{abstract}

Keywords: categorization, text mining, youtube.

\section{Introdução}

O Youtube, uma plataforma criada por Chad Hurley, Steve Chen e Jawed Karim, foi lançado no final de junho de 2005 com o intuito de compartilhar vídeos na Internet. Segundo Burgess e Green (2009), possui uma interface simples e intuitiva, onde os usuários podem, sem elevado conhecimento técnico, assistir e publicar vídeos.

A plataforma está voltada para vídeos e possui conteúdos sobre diversas áreas do conhecimento. Segundo Heilbron (2019), a cada minuto são enviadas 400 horas de vídeos, e mais de 2 bilhões de usuários acessam a plataforma mensalmente (YOUTUBE, 2019b). Segundo Youtube (2017), 1,5 bilhões de pessoas conectadas pelo mundo acessam o Youtube mensalmente e $95 \%$ da população brasileira conectada acessa a plataforma 
ao menos uma vez por mês. No Brasil, 9 em cada 10 usuários do Youtube acessam a plataforma com o intuito de aprender algo novo e mais da metade acredita que o Youtube é o lugar onde encontra-se tudo o que se deseja ver e aprender (YOUTUBE, 2019a).

O Youtube/Google em parceria com a fundação Lemann desenvolveram um canal denominado Youtube Edu com a proposta de fornecer conteúdos educacionais gratuitos e de qualidade, em português. O conteúdo do canal é voltado para o Ensino Fundamental e Ensino Médio, e aborda temas das disciplinas de Biologia, Física, Química, Geografia, História, Língua Espanhola, Língua Inglesa, Língua Portuguesa, e Matemática. O canal possui vídeos e playlists segmentadas por curso e série, além de "aulões" para o Enem.

Apesar de apresentar diversos materiais sobre as áreas mencionadas, o conteúdo do Youtube Edu é limitado no sentido de que não contempla temáticas de cursos técnicos e de nível superior. Observa-se, adicionalmente, que, por ser um canal, o Youtube Edu é englobado pelo Youtube, ou seja, uma pesquisa no Youtube pode retornar vídeos do canal Youtube Edu, contudo, o inverso não é verdadeiro. Este é o motivo desta pesquisa se basear na utilização da plataforma Youtube e não no canal Youtube Edu.

O Youtube, sob o ponto de vista educacional, pode ser entendido como um repositório de Objetos de Aprendizagem (OA). De forma geral, os OA podem ser definidos como "qualquer recurso digital que possa ser reutilizado com o intuito de auxiliar na educação, desde que possa ser entregue pela rede, como imagens, vídeos, animações, textos e outros" (WILEY, 2000).

Neste sentido, a oferta de OA é realizada pela plataforma por meio do seu mecanismo de busca, i.e., busca-se por um conteúdo e a plataforma apresenta os OA condizentes à expressão de pesquisa utilizada. Essa "assistência" faz parte da área de estudos que aborda a Recomendação de Objetos de Aprendizagem. Segundo Pontes et al. (2014), a Recomendação de Objetos de Aprendizagem é utilizada para identificar usuários, armazenar suas preferências e recomendar itens que podem ser produtos, serviços e/ou conteúdos, de acordo com suas necessidades e interesses.

Apesar de disponibilizar conteúdos sobre as mais diversas áreas e possuir um extenso acervo de vídeos, é possível identificar um problema que se relaciona aos resultados retornados pela plataforma ao se realizar uma pesquisa, pois, em muitos casos, o número de resultados retornado é muito elevado, sendo muitos destes de baixa qualidade e/ou não muito relacionados à pesquisa realizada.

Uma das formas de se reduzir o número de resultados da pesquisa e de, portanto, particularizar o retorno da mesma, se faz pela especificação da categoria dos vídeos pelos quais se busca. De fato, o Youtube categoriza seus vídeos, porém, não é possível realizar pesquisas por elas, e a maioria dos usuários não tem conhecimento sobre essa categorização.

No intuito de se utilizar as categorias dos vídeos para a pesquisa na plataforma e consequentemente refinar o conteúdo retornado, Carvalho et al. (2020a) desenvolveram um sistema Web que permitiu a realização de pesquisa de vídeos por categorias. Entretanto, os resultados apontam que muitas vezes a categorização dos vídeos não é relevante ou que as categorias estão erroneamente atribuídas aos vídeos, o que prejudica ainda mais a a qualidade da pesquisa. Neste sentido, apontou-se que não se deve considerar as categorias dos vídeos do Youtube como determinantes para a identificação dos mesmos (CARVALHO et al., 2020b). 
Este trabalho propõe uma nova forma de categorização de vídeos do Youtube por meio dos comentários postados pelos usuários. Neste sentido, analisam-se os comentários de diversos vídeos educacionais e não educacionais da plataforma. Os comentários são processados para a obtenção dos vocábulos e termos mais frequentes, com o intuito de identificar se os comentários podem ser utilizados para a categorização de vídeos educacionais.

Resultados preliminares demonstram diferenças entre os termos e vocábulos mais frequentes empregados nos vídeos educacionais e não educacionais. Observa-se, por exemplo, que termos como "melhor professor", e "ótima aula" figuram frequentemente em comentários de vídeos educacionais, mas não ocorrem em comentários de vídeos não educacionais. De forma semelhante, verificou-se que os radicais "profes", "aul", e "obrig" figuram frequentemente em comentários de vídeos vídeos educacionais. Neste sentido, aponta-se que os comentários possuem potencial para serem utilizados com o intuito de categorizar os vídeos educacionais do Youtube.

O presente trabalho se organiza como segue. A seção 2 apresenta os principais trabalhos relacionados a presente pesquisa. A seção 3 descreve a metodologia experimental utilizada. A seção 4 discute os resultados obtidos. Por fim, a seção 5 apresenta as considerações finais e apontamentos a trabalhos futuros.

\section{Trabalhos Relacionados}

Esta seção aborda os trabalhos relacionados a esta pesquisa. Destaca-se que poucos trabalhos foram identificados referentes a categorização de vídeos educacionais do Youtube. Neste sentido, esta seção relaciona os trabalhos que abordam o tema de Recomendação de OA utilizando a Wikipédia e o Youtube, por se tratarem de pesquisas que mais se aproximam do problema abordado.

Menolli et al. (2011) objetivam gerar OA com a Web 2.0, através da Wikipedia, utilizando tecnologias semânticas e o padrão Learning Object Metadata (LOM). Em sua proposta, acessam os conteúdos inseridos na plataforma, e realizam a mineração de textos a fim de extrair e classificar os conteúdos de acordo com o padrão LOM. Com a utilização desse padrão, encontram os atributos e metadados da página, gerando um XML-schema com os metadados da página em questão. Concluem que essa abordagem é necessária, pois facilita a utilização dos conteúdos devido as ferramentas wikis não considerarem como o conteúdo será utilizado.

Abu-El-Haija et al. (2016) abordam a classificação de vídeos do Youtube com o objetivo de desenvolver um sistema de multi-classificação. A base de dados utilizada possui aproximadamente 8 milhões de vídeos englobando um total de 1,9 bilhão de quadros, e 500 mil horas de conteúdos. A pesquisa é realizada em duas etapas, a saber: 1) os rótulos dos vídeos são obtidos por meio do Knowledge Graph entities; 2) os vídeos são processados frame a frame, e categorizados por uma Rede Neural Convolucional pré-treinada no ImageNet. O ImageNet é um banco de dados visual com diversos objetos/entidades já classificados. Por meio do processamento de mais de 50 anos de vídeos, provendo 2 bilhões de frames, e mais de 8 milhões de vídeos que podem ser modelados rapidamente em uma única máquina, o trabalho aponta sua contribuição no sentido de auxiliar o desenvolvimento de pesquisas sobre compreensão de vídeos. 
Apesar da categorização em diversas classes, não foi encontrada uma categoria específica para vídeos educacionais. O trabalho cita a categoria "Jobs \& Education" e nela estão universidades, salas de aulas, palestras, etc. Desta forma, um vídeo que possua imagens de um campus universitário, por exemplo, será enquadrado nessa categoria, e não necessariamente é um vídeo educacional.

Júnior e Dorça (2018) apresentam uma abordagem para criação e recomendação de OA por meio da plataforma Wikipédia. A abordagem é definida por três etapas, a saber: 1) enriquecimento da ontologia por meio dos metadados das seções wiki; 2) recomendação dos OA tratada como um Problema de Cobertura de Conjuntos, com a utilização de Algoritmo Genético; 3) uso de operações CRUD (Create, Read, Update, Delete). $\mathrm{O}$ trabalho conclui que a abordagem adotada resolve o problema da recomendação de OAs, retornando soluções de elevada qualidade.

Pinheiro et al. (2018) apresentam o Easy Youtube, um Sistema de Recomendação de OA baseado no Youtube. O funcionamento do sistema se faz em seis etapas, a saber: 1) enriquecimento de consultas - definição de temas pré-definidos, cadastrados por especialistas; 2) extração de vídeos - busca de vídeos, que pode ser realizada por meio de pesquisa ou de temas pré-definidos; 3) pré-processamento - tratamento dos textos (em português), com remoção de pontuação, espaços, etc.; 4) classificação - utilização de um algoritmo para classificar os vídeos considerados como educacionais e de qualidade; 5) engenho de recomendação - o sistema recebe os vídeos considerados "bons" e classifica-os; 6) coletor de feedback - o usuário avalia a recomendação fornecida pelo sistema por meio de notas, que variam de 1 a 7 estrelas. O trabalho aponta suas principais contribuições nos pontos: 1) o Sistema de Recomendação desenvolvido pode ser utilizado como solução para vários domínios de aplicação; 2) o sistema serviu como prova de conceito para melhorar as recomendações, por meio de características do Youtube, como a avaliações dos usuários, e linguagem nativa do vídeo.

O trabalho apresentado não detalha partes importantes de pesquisa. Por exemplo, para a classificação de vídeos considerados de qualidade, afirma-se que utilizou-se um conjunto de treinamento de 100 vídeos, contendo avaliações realizadas por especialistas e alunos que já tiveram em sua grade curricular o assunto "Orientação a Objetos/Herança". Porém, não se explica de que maneira esta análise foi realizada, e quais características dos vídeos foram consideradas. Outro ponto de questionamento, é o apontamento de que, devido ao prazo para a realização da pesquisa, o trabalho focou em "algumas características para o experimento". Tais características não foram descritas.

Thelwall (2018) analisa os comentários de vídeos do Youtube relacionados a estilos de dança. A base de dados utilizada contém 36.702 vídeos. O trabalho objetiva identificar, por meio dos comentários postados nos vídeos da plataforma, os tipos de dança, relações quanto aos gêneros (masculino e feminino), sentimentos expressados, e discussões referente aos estilos. Utiliza-se, para tanto, o método denominado Comment Term Frequency Comparison (CTFC) na tentativa de identificação de subtópicos/subtemas das discussões sobre um tópico nos comentários do Youtube, questões de gênero, sentimentos, e relacionamento entre tópicos. O método utilizado define com sucesso diversas atitudes predominantes em homens e mulheres. Os 10 termos homem-associados foram: shit, fuck, shuffle, man, fucking, crip, dude, bro, shuffling, hardstyle. Por sua vez, os 10 termos mulher-associados foram: she, amazing, her, beautiful, cute, omg, belly, ballet, really, workout. A análise de sentimentos forneceu ideias plausíveis dos motivos pelos quais as danças eram apreciadas. Os 10 termos positivos mais utilizados foram: please, nice, wow, 
beautiful, loved, job (e.g. nice/great/good job), pretty, hope, perfect, keep (going/up the good work/it up). Por sua vez, os 10 termos negativos mais utilizados foram: shit, fuck, killed, stupid, wtf, hate, idiot, dislike, die, dead.

Os autores afirmam que os resultados podem servir como ponto de partida para análises mais aprofundadas sobre o tema, e que a pesquisa destacou as diferenças de gêneros, sentimentos, e subtópicos entre as danças. Também apontam que método utilizado pode ser útil para discutir, em larga escala, fenômenos específicos do Youtube, como também pode ser útil em outros contextos para fornecer uma análise exploratória inicial de um problema que não havia sido pesquisado anteriormente.

Carvalho et al. (2020b) realizam uma análise dos vídeos e das categorias utilizadas pela plataforma Youtube. Aponta-se que a plataforma apresenta 3 pontos críticos quanto ao seu mecanismo de busca e sua categorização, a saber: 1) falta de transparência quanto à categorização de um vídeo; 2) informações errôneas quanto à categorização dos vídeos, e 3) impossibilidade de se pesquisar vídeos por suas categorias. Devido a esses pontos críticos, o trabalho conclui que a categorização realizada pela plataforma não pode ser determinante para a identificação dos vídeos. Ademais, sugerem-se que novas abordagens são necessárias para categorização de vídeos educacionais.

\section{Metodologia}

A metodologia adotada para o desenvolvimento do presente trabalho é apresentada na figura 1 e detalhada a seguir.

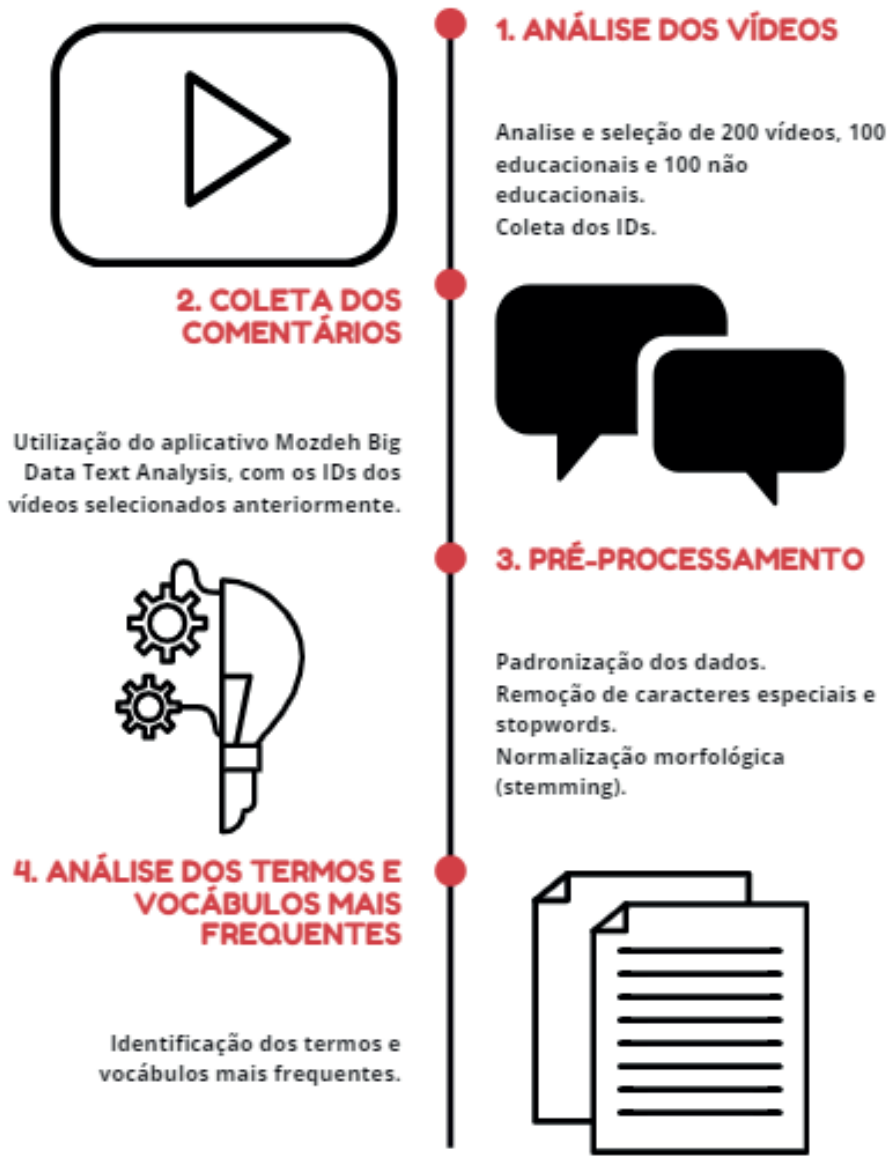

Figura 1. Metodologia utilizada. 
1. Análise dos vídeos. Esta etapa consistiu na identificação manual (por meio de visualização) de vídeos educacionais e não educacionais da plataforma.

Para a análise dos vídeos, adotou-se a definição de vídeos educacionais proposta por (GOMES, 2008). Segundo o autor, um vídeo educativo é um "produto específico, produzido com intenção didático-pedagógica e que considera seu contexto de recepção como especialmente a escola e a sala de aula, sendo, portanto, intrinsecamente diferente dos vídeos de documentários, entrevistas, reportagens, etc.”. Adotou-se tal definição, pois, acredita-se, que a mesma se mostra abrangente o suficiente para a análise proposta neste trabalho, ao mesmo tempo em que pode ser utilizada para a categorização de um vídeo como educacional ou não.

Nesta etapa foram analisados 200 vídeos, sendo 100 educacionais, e 100 não educacionais. Esse número foi selecionado como ponto de partida para a possível identificação de um vocabulário específico nos comentários de vídeos educacionais do Youtube.

2. Coleta dos comentários. Esta etapa consistiu em armazenar os identificadores (IDs) dos vídeos selecionados na etapa anterior, e coletar os comentários utilizando o aplicativo Mozdeh Big Data Text Analysis, também utilizado em (THELWALL, 2018). O aplicativo foi utilizado apenas para a coleta dos comentários, pois apesar de possuir outras funcionalidades, estas são específicas para vocábulos em inglês.

3. Pré-processamento dos comentários. Esta etapa consistiu nas seguintes subetapas, a saber.

- Padronização dos dados: transformação de todas as letras em maiúsculo para minúsculo.

- Remoção de caracteres especiais e stopwords: no primeiro momento foram removidos os caracteres especiais como: !, \#, @, dentre outros. Isso é necessário, por exemplo, para que comentários tais como, "muito bom!" e "muito bom"; "melhor professor!" e "melhor professor", sejam considerados equivalentes. As stopwords podem ser consideradas palavras não relevantes para o texto. Estas podem ser artigos, preposições, advérbios, pronomes, e outras palavras auxiliares (MORAIS; AMBRÓSIO, 2007). Alguns exemplos de stopwords são "tem", "isto", "aos", dentre outras.

- Normalização morfológica (stemming): consiste em remover os prefixos e os sufixos dos vocábulos, mantendo apenas o radical da palavra, ou seja, fazendo com que palavras como "professor" e "professora", sejam "reduzidas"a "profes", por exemplo.

4. Análise dos termos e vocábulos mais frequentes. Esta etapa consistiu na identificação dos termos e vocábulos mais frequentes nos comentários. Tal passo possibilitou a análise dos comentários para a identificação das diferenças entre os comentários presentes nos vídeos educacionais e não educacionais.

\section{Resultados}

A base de dados é composta por 100 vídeos educacionais e 100 vídeos não educacionais. Os resultados dos termos mais frequentes nos comentários dos vídeos são apresentados na tabela 1. Por sua vez, a tabela 2 apresenta os vocábulos mais frequentes nos comentários dos vídeos.

Considerandos os vídeos educacionais, têm-se um total de 77.095 comentários, e 1.002.070 vocábulos. Já os vídeos não educacionais contêm 81.464 comentários e 
Tabela 1. Termos mais frequentes nos comentários dos vídeos.

\begin{tabular}{|c|c|c|c|}
\hline \multicolumn{4}{|c|}{ Termos mais frequentes } \\
\hline \multicolumn{2}{|c|}{ Educacional } & Não Educacional \\
\hline Termo & Quantidade & Termo & Quantidade \\
\hline muito bom & 572 & top & 152 \\
\hline melhor professor & 291 & oi & 117 \\
\hline ótima aula & 138 & primeiro & 97 \\
\hline obrigado & 127 & ah é & 95 \\
\hline amei & 126 & muito bom & 91 \\
\hline eu & 113 & eu & 61 \\
\hline show & 103 & salve & 58 \\
\hline
\end{tabular}

Tabela 2. Vocábulos mais frequentes nos comentários dos vídeos.

\begin{tabular}{|c|c|c|c|}
\hline \multicolumn{4}{|c|}{ Vocábulos mais frequentes } \\
\hline \multicolumn{2}{|c|}{ Educacional } & Não Educacional \\
\hline Vocábulo & Quantidade & Vocábulo & Quantidade \\
\hline profes & 16421 & pra & 6363 \\
\hline aul & 11828 & faz & 5666 \\
\hline obrig & 7544 & músic & 4020 \\
\hline melhor & 6183 & vai & 3723 \\
\hline ajud & 6087 & tod & 3658 \\
\hline vc & 5073 & melhor & 3411 \\
\hline aprend & 4974 & vc & 3365 \\
\hline
\end{tabular}

\subsubsection{3 vocábulos.}

Observa-se que os vídeos não educacionais apresentam termos como risadas, que não foram incluídos na tabela 1 por serem irrelevantes ao estudo. Neste sentido, apontamse que os termos "kkkkk" (cinco k's) e "kkkkkk" (seis k's), figuram, respectivamente, 65 e 59 vezes, e ocupariam, respectivamente, a sexta e oitava posição.

Ao analisar a tabela 1, percebem-se as diferenças entre os termos mais frequentes. O único termo utilizado nos comentários de ambas as classes de vídeos é "muito bom", sendo utilizado 572 vezes nos cometários dos vídeos educacionais, e 91 vezes nos comentários dos vídeos não educacionais. Apesar de estar presente nos comentários de ambas as classes de vídeos, o número de vezes em que este termo foi utilizado é bastante diferente: mais de 6 vezes nos comentários dos vídeos educacionais quando comparada à utilização nos comentários dos vídeos não educacionais.

Outras palavras como "amei", "eu" e "show", apesar de figurarem mais frequentemente nos comentários dos vídeos educacionais, podem não ser muito indicadas para a utilização para a classificação, por serem muito genéricas. Já os termos como "melhor professor" e "ótima aula", por serem mais específicos ao contexto educacional, podem ser indicados para a categorização de um vídeo.

$\mathrm{Na}$ tabela 2, percebe-se que as únicas palavras que figuram tanto nos comentários dos vídeos educacionais quanto nos cometários dos vídeos não educacionais são "melhor" e "vc". Contudo, mesmo presente em ambas as classes de vídeos, o número de vezes em que figuram é muito diferente: "melhor" foi utilizado 6183 vezes, e "vc" 5073 vezes nos 
comentários dos vídeos educacionais, em comparação a, respectivamente, 3411 vezes e 3365 vezes nos comentários dos vídeos não educacionais.

Os radicais "profes", "aul”, "obrig", "ajud", e "aprend" figuram apenas nos comentários dos vídeos educacionais, sendo, portanto, potencialmente adequados para serem utilizados para a classificação de um vídeo.

Devido as marcantes diferenças entres os vocábulos e os termos mais frequentes nos comentários de vídeos educacionais e não educacionais, aponta-se que o uso dos comentários é uma possível alternativa para a categorização dos vídeos educacionais da plataforma.

\section{Considerações Finais e Trabalhos Futuros}

Este trabalho demonstrou que existem diferenças fundamentais entre os vocabulários dos comentários de vídeos educacionais e não educacionais do Youtube. Pôde-se identificar grandes variações nos termos mais frequentes e nas palavras mais utilizadas, demonstrando que os comentários apresentam grande potencial para serem utilizados para categorizar os vídeos educacionais da plataforma.

A continuidade dessa pesquisa se desenvolve em duas frentes, saber: 1) utilização de técnicas de Aprendizagem de Máquina para processar os comentários dos vídeos e classificá-los como educacionais ou não educacionais; 2) desenvolvimento de um sistema Web que utiliza os metadados e os comentários dos vídeos para categorizá-los, bem como para mensurar o nível de qualidade dos mesmos.

\section{Referências}

ABU-EL-HAIJA, S.; KOTHARI, N.; LEE, J.; NATSEV, P.; TODERICI, G.; VARADARAJAN, B.; VIJAYANARASIMHAN, S. Youtube-8m: A large-scale video classification benchmark. arXiv preprint arXiv:1609.08675, 2016.

BURGESS, J.; GREEN, J. YouTube e a revolução digital. [S.1.: s.n.], 2009. 24 p.

CARVALHO, H. C. F. B.; PITANGUI, C. G.; ASSIS, L.; VIVAS, A. Educavídeos: Um sistema de recomendação de objetos de aprendizagem de vídeos educacionais do youtube. In: CONGRESSO BRASILEIRO DE ENSINO SUPERIOR A DISTÂNCIA, XVII., 2020, Goiânia. Anais. Goiânia, 2020.

CARVALHO, H. C. F. B.; PITANGUI, C. G.; TRINDADE, E. A.; ASSIS, L.; VIVAS, A. Learning objects and youtube: an analysis of videos and their categories. In: LATIN AMERICAN CONFERENCE ON LEARNING TECHNOLOGIES, XV., 2020, Loja. Anais. Loja, 2020.

GOMES, L. Vídeos didáticos: uma proposta de critérios para análise. Revista Brasileira de Estudos Pedagógicos, v. 89, n. 223, 2008.

HEILBRON, F. D. C. Understanding Human Activities at Large Scale. Tese (Doutorado), 2019.

JÚNIOR, C. B.; DORÇA, F. Uma Abordagem para a Criação e Recomendação de Objetos de Aprendizagem usando um Algoritmo Genético, Tecnologias da Web Semântica e uma Ontologia. 2018. 1533 p.

MENOLLI, A.; MALUCELLI, A.; REINEHR, S. Criaçao semi-automatica de objetos de aprendizagem a partir de conteúdos da wiki. In: BRAZILIAN SYMPOSIUM ON 
COMPUTERS IN EDUCATION (SIMPÓSIO BRASILEIRO DE INFORMÁTICA NA EDUCAÇÃO-SBIE), XXII., 2011, Aracaju. Anais. Aracaju, 2011.

MORAIS, E. A. M.; AMBRÓSIO, A. P. L. Mineração de textos. Relatório Técnico-Instituto de Informática (UFG), 2007.

PINHEIRO, R. R. A. et al. Sistema de recomendação de vídeos educacionais: um estudo de caso no you tube. Universidade Federal de Alagoas, 2018.

PONTES, W. L.; FRANÇA, R. M.; COSTA, A. P. M.; BEHAR, P. Filtragens de Recomendação de Objetos de Aprendizagem: uma revisão sistemática do CBIE. 2014. $549 \mathrm{p}$.

THELWALL, M. Social media analytics for youtube comments: Potential and limitations. International Journal of Social Research Methodology, Taylor \& Francis, v. 21, n. 3, p. 303-316, 2018.

WILEY, D. A. Learning object design and sequencing theory. Tese (Doutorado) Brigham Young University, 2000.

YOUTUBE. Youtube Insights 2017. 2017. Acesso em: 16 de Abril de 2019. Disponível em: 〈https://www.thinkwithgoogle.com/intl/pt-br/youtubeinsights/2017/introducao/〉.

YOUTUBE. YouTube Insights. 2019. Acesso em: 17 de Abril de 2019. Disponível em: 〈https://youtubeinsights.withgoogle.com〉.

YOUTUBE. Youtube para imprensa. 2019. Acesso em: 16 de Abril de 2019. Disponível em: 〈https://www.youtube.com/intl/pt-BR/yt/about/press/〉. 